\title{
Correction to: The role of using different reference population in the prevalence of low BMD in the thalassemia patients
}

\section{Mohammad Reza Mohajeri-Tehrani ${ }^{1}$ - Najmeh Darvishian ${ }^{1}$ - Faezeh Arab ${ }^{1} \cdot$ Sedigheh Salemkar $^{1} \cdot$ Fariba Mohseni $^{1}$. Bagher Larijani ${ }^{1} \cdot$ Zohreh Hamidi $^{1}$}

Published online: 23 December 2019

(C) Springer Nature Switzerland AG 2019

\section{Correction to: Journal of Diabetes \& Metabolic Disorders} https://doi.org/10.1007/s40200-019-00455-6

The article 'The role of using different reference population in the prevalence of low BMD in the thalassemia patients', written by Mohammad Reza Mohajeri-Tehrani, Najmeh Darvishian, Faezeh Arab, Sedigheh Salemkar, Fariba Mohseni, Bagher Larijani and Zohreh Hamidi, was originally published electronically on the publisher's internet portal (currently SpringerLink) on 14 November 2019 with open access. With the author(s)' decision to step back from Open Choice, the copyright of the article changed on 24 December 2019 to (C) Springer Nature Switzerland AG 2019 and the article is forthwith distributed under the terms of copyright.

The online version of the original article can be found at https://doi.org/ 10.1007/s40200-019-00455-6

Zohreh Hamidi

zohreh.hamidi@gmail.com

1 Endocrinology and Metabolism Research Center, Endocrinology and Metabolism Clinical Sciences Institute, Tehran University of Medical Sciences, Shariati Hospital, 5th floor, North Kargar Ave. P.C.,

Tehran 14114-13137, Iran 